a trained musculoskeletal radiologist. For each DECT, clinical and biochemical characteristics of each patient were collected retrospectively.

Results: 22 patients (men 77\%), mean age 62.5 years and mean BMI 28.4 $\mathrm{kg} / \mathrm{m}^{2}$ were included. Mean gout duration was $108.0 \pm 114.4$ months, mean of last available serum uric acid level was $520 \pm 193 \mu \mathrm{mol} / \mathrm{l}$, and 15 patients had at least one clinical tophus. Mean estimated glomerular filtration rate (MDRD formula) was $47 \pm 27 \mathrm{ml} / \mathrm{min} / 1.73 \mathrm{~m}^{2}$. One patient was on hemodialysis and one had received kidney transplant.

A total of 39 DECT has been performed: 28 of peripheral joints and 11 of the spine (9 lumbar, 1 sacroiliac and 1 cervical). Spinal DECT were done in 10 patients to explore recurrent inflammatory pain $(n=3$ lumbar, 1 cervical and 1 buttock) or mechanical back pain ( $n=2$ lumbar). 4 spinal DECT were performed in asymptomatic patients with extended peripheral tophi. Spinal MSU crystal deposits were disclosed by DECT in $83 \%(5 / 6)$ and $25 \%$ (1/4) of symptomatic and asymptomatic patients, respectively. In all painful patients, MSU crystal deposition was considered as a likely explanation of spinal symptoms. MSU crystal depositions was identified in apophyseal joints $(n=5)$, cervical intervertebral disc $(n=1)$ and yellow or interspinous ligaments $(n=4)$. All involved apophyseal joints were eroded (figure 1). No vertebral bone erosion was observed. Calcification of spinal tophus was observed in 4 patients. DECT identified peripheral deposits in $15 / 18(83.3 \%)$ patients. In peripheral DECT, bone erosions were observed in $71.4 \%$ and joint effusion in $32.1 \%$ of DECT positive peripheral joints. MSU crystal depositions were observed in tendons, cartilages or synovial membranes in $82.1 \%$ of positive DECT joints and in soft tissues in $64.3 \%$ of positive patients. MSU crystal deposits were calcified in 7 cases.

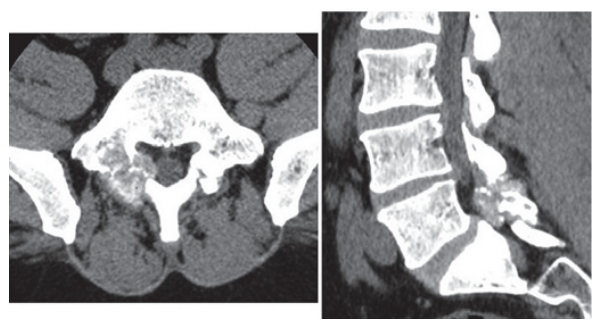

Conclusions: MSU crystal depositions at the spine are present in $60 \%$ of patients in this retrospective DECT study. DECT can represent a performing imaging procedure for their detection in symptomatic patients. Further studies are needed to assess the clinical utility of DECT of the spine in gout.

References:

[1] Janssens HJ, Fransen J, van de Lisdonk EH, et al. A diagnostic rule for acute gouty arthritis in primary care without joint fluid analysis. Arch Intern Med 2010;170:1120-6.

Disclosure of Interest: None declared

DOI: 10.1136/annrheumdis-2017-eular.6835

\section{THU0425 THE FIRST METATARSOPHALANGEAL JOINT (MTP1) IS NOT THE MAIN LOCALIZATION OF GOUT AT DIAGNOSIS IN SUB-SAHARAN AFRICA}

F. Kemta Lekpa, M.S. Doualla, H. Namme Luma. Service de Médecine Interne, Douala General Hospital, Douala, Cameroon

Background: Numerous data in sub-Saharan Africa suggest that MTP1 is not the hallmark of gout (1-5).

Objectives: We carried out this study with the aim to determine the joint most involved at the time of diagnosis of gout in Cameroon.

Methods: We performed a cross-sectional study in all outpatients seen at the Rheumatology unit of the Douala General Hospital, Cameroon, between 2004 and 2014. We included patients with diagnosis of gout according to ACR criteria 1977.

The main characteristics of gout at diagnosis were collected, particularly the joints involved.

A $p<0.05$ was significant.

Results: At the end of this study, 511 patients ( 415 men and 96 women) with the diagnosis of gout were included. The mean age was $55.9 \pm 10.8$ years.

Joint pain $(n=508,99.4 \%)$ was the leading reason for consultation at the time of diagnosis. The knees $(n=300,62.6 \%))$, ankles $(n=187,39.0 \%)$ and MTP1 $(n=128$, $26.7 \%$ ) were the most affected joints. Table 1 presents the frequency of the joints affected, comparing our results with those of the other African series.

There was no difference between MTP1 and others joints location (particularly knees and ankles) according to age, sex, place of residence, duration of disease, uric acid level, and associated comorbidities ( $p>0.05$ ).

Conclusions: MTP1 is not the joint most involved at the time of diagnosis of gout in sub-Saharan Africa. Diagnosis of gout should be considered before any inflammatory knee and ankle pain in patients from sub-Saharan Africa. Genetic studies would provide a better understanding of this feature.

\section{References:}

[1] Kodio B, et al. Afr J Rheumatol. 2015 3(1): 22-26.

[2] Adelewo OO, et al. Afr J Rheumatol. 2014 1(2): 23-28.

[3] Singwé-Ngandeu M, et al. Mali Medical. 2009 24:17-20.
Table 1. Comparison of the most affected joints of the different African series*

\begin{tabular}{lcccccc}
\hline & $\begin{array}{c}\text { Our study } \\
\text { (Cameroon), } \\
2017\end{array}$ & $\begin{array}{c}\text { Kodio } \\
\text { (Mali), }\end{array}$ & $\begin{array}{c}\text { Adelowo } \\
\text { (Nigeria), }\end{array}$ & $\begin{array}{c}\text { Singwé- } \\
\text { Ngandeu } \\
\text { (Cameroon), } \\
2015\end{array}$ & $\begin{array}{c}\text { Mijiyawa } \\
\text { (Togo), } \\
2000\end{array}$ & $\begin{array}{c}\text { Cassim } \\
\text { (South Africa), } \\
1994\end{array}$ \\
\hline Number of patients & 511 & 100 & 146 & 139 & 160 & 107 \\
Sex (M/F) & $415 / 96$ & $45 / 55$ & $108 / 38$ & $131 / 8$ & $159 / 1$ & $93 / 14$ \\
Mean age & $55.9 \pm 11$ & 57 & $53.4 \pm 11$ & $55.7 \pm 10$ & 44 & $50.5 \pm 11.5$ \\
Joints involved (tender and/or stiffness), $\mathrm{n}(\%)$ & & & & \\
Knees & $300(62.6)$ & $92(92 \%)$ & $81(55.5)$ & $60(43.2)$ & $82(51.2)$ & $91(85)$ \\
Ankles & $187(39.0)$ & $45(45 \%)$ & $50(34.2)$ & $67(48.2)$ & $90(56.2)$ & $66(61.7)$ \\
1st MTP joint & $128(26.7)$ & $22(22 \%)$ & $21(14.4)$ & $65(46.8)$ & $100(62.5)$ & $80(74.8)$ \\
Wrists & $93(19.4)$ & $20(20 \%)$ & $14(9.6)$ & $30(21.6)$ & $30(18.7)$ & - \\
Elbows & $81(16.9)$ & $14(14 \%)$ & $12(8.2)$ & $20(10.7)$ & $35(21.9)$ & - \\
PIP joints & $56(11.7)$ & - & $13(8.9)^{\ddagger}$ & - & $15(9.4)^{\dagger}$ & - \\
MCP joints & $53(11.1)$ & - & - & - & - & - \\
Tarsal region & $50(10.4)$ & - & - & - & $50(31.2)$ & - \\
Shoulders & $49(10.2)$ & $6(6 \%)$ & - & - & - & - \\
Others MTP joints & $24(5.0)$ & - & - & - & $15(9.4)$ & - \\
DIP joints & $15(3.1)$ & - & - & - & - & - \\
Others & $30(6.3)$ & $1(1 \%)$ & - & $13(9.6)$ & $9(5.6)$ & - \\
\hline
\end{tabular}

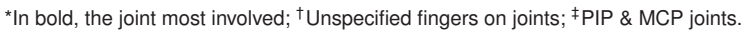

[4] Mijiyawa M, et al. Joint Bone Spine. 2000;67(5):441-5.

[5] Cassim B, et al. Ann Rheum Dis. 1994 Nov;53(11):759-62.

Disclosure of Interest: None declared

DOI: 10.1136/annrheumdis-2017-eular.6799

\section{THU0426 LONG-TERM ADHERENCE TO URATE-LOWERING THERAPY IN GOUT: DO NOT BLAME ON THE PATIENTS}

F. Perez-Ruiz, I. Urionagüena, S.P. Chinchilla. Rheumatology Division, Hospital Universitario Cruces and Biocruces Health Research Institute, Baracaldo, Spain

Background: adherence to urate-lowering treatment (ULT) in patients with gout is reported to be lower than $50 \%$ in the first year, below $20 \%$ at 2-year, and worse than in other chronic conditions such as hypertension, diabetes, or hyperlipidemia. Objectives: to evaluate adherence to ULT both overall and during follow-up, to compare it to the adherence to medications for associated comorbidities, and to explore potential causes for non-adherence to ULT.

Methods: transversal study of a nested cohort of patients in a gout clinic in the hospital setting who were scheduled for a follow-up visit during 6 consecutive months in 2016. General data of patients, along with variables related to gout and to comorbid conditions are systematically retrieved at first visit; prescribed ULT, doses, adherence, and serum urate levels were obtained during the follow-up visits. Adherence was retrieved as medication possession rate (MPR) according to pharmacy offices from government electronic databases (including $>98 \%$ of the general population). Also, MPRs of drugs prescribed for hypertension, diabetes (only oral), and hyperlipidemia were obtained; if more than one drug prescribed for any of the previous, the best adherence per comorbidity treatment was entered. Good adherence was considered as MPR $>80$ percent of that prescribed, target serum urate $(\mathrm{s} \cup A)$ as $<0.36 \mathrm{mmol} / \mathrm{L}$. Patients are educated at first visit and encouraged to be adherent from baseline through follow-up visits.

Results: adherence data were available from 209 patients who were scheduled for a follow-up visit during the observation period; $14(6.7 \%)$ patients did not atend the visit. This sample was formed by $90 \%$ male, only $55 \%$ had received ULT previous to first visit, median age was 65 years at follow-up visit, $47 \%$ and $44 \%$ showed poliarticular and tophaceous disease at baseline, respectively. MPR overall showed a median of $89 \%$ (IQR 79-94, N=209) for ULT (72\% had MPR $>80$ ), and $89 \%$ (IQR 81-94, $\mathrm{N}=119$ ), 88\% (IQR 79-94, $\mathrm{N}=65$ ), and $82 \%$ (IQR 77-93, N=28) for hypertension, hyperlipidemia, and diabetes respectively ( $<<0.05$ only for diabetes).

Adherence was lower for patients who did not attend the scheduled visit (median MPR $58 \%$ vs. $86 \%$, MPR $>80,21 \%$ vs. $75 \%, p<0.01)$. Adherence was lower during the first year $(80 \%, N=67)$ compared to 2 nd and 3 rd year period $(86 \%$, $\mathrm{N}=67$ ) or 4 year or over ( $89 \%, \mathrm{~N}=75)$. MPR $>80$ was $57 \%, 76 \%$ y $81 \%$ for the same periods of follow-up, respectively. Good adherence was associated to a rate of target serum urate of $90 \%$, compared to $72 \%$ for patients showing MPR $<80$. Male gender and un-attendance to scheduled visit were statistically associated to $\mathrm{MPR}<80$ in multivariate analysis, and numerically to increasing age and overall comorbidity.

Conclusions: adherence to ULT measured as MPRs in a cohort of educated patients is good, sustained during follow-up, and similarly good to that for comorbid conditions (hypertension, hyperlipidemia, and diabetes); therefore, we cannot blame poor adherence on the patients anymore. Targeting absenteeism could be an opportunity for further improvement.

Disclosure of Interest: F. Perez-Ruiz Consultant for: Amgen, Ardea, AstraZeneca, Gruenenthal, Menarini, Speakers bureau: AstraZeneca, Gruenenthal, Menarini, I. Urionagüena: None declared, S. Chinchilla: None declared DOI: 10.1136/annrheumdis-2017-eular.2994 